\title{
Pacific
}

Journal of

Mathematics

\section{TENT SPACES OVER GENERAL APPROACH REGIONS AND} POINTWISE ESTIMATES

\author{
MARÍA J. CARRO AND JAVIER SORIA
}




\title{
TENT SPACES OVER GENERAL APPROACH REGIONS AND POINTWISE ESTIMATES
}

\author{
María J. Carro and Javier Soria
}

\begin{abstract}
We consider the study of the tent spaces over general (possibly tangential) approach regions and their atomic decomposition. As a consequence, we obtain some pointwise estimates for a class of operators, using the duality properties of a certain type of Carleson measures. In particular, we can get the boundedness of a family of bilinear operators defined on the product of $L^{q}$ and some space of measures, into a Lipschitz space; we give yet another proof of the pointwise boundedness for the Fourier transform of distributions in $H^{p}$ and we improve and generalize the Fejjer-Riesz inequality for harmonic extensions of $H^{p}$ functions.
\end{abstract}

Several authors have studied the boundedness of maximal operators defined by means of general subsets. For example, in [8], a Hardy-Littlewood type operator is associated with a collection of subsets $\Omega_{x} \subset \mathbf{R}_{+}^{\mathbf{n}+1}, x \in \mathbf{R}^{\mathbf{n}}$. The natural way to define the balls for these sets is to take the subset of $\Omega_{x}$ at level $t$, that is, the set of points $z \in \mathbf{R}^{\mathbf{n}}$ so that $(z, t) \in \Omega_{x}$. Our idea is to also replace the cone $\Gamma(x)=\left\{(y, t) \in \mathbf{R}_{+}^{\mathbf{n}+1}:|x-y|<t\right\}$ in the definition of the tent spaces (see [2]), by a more general family of subsets of $\mathbf{R}_{+}^{\mathbf{n}+1}$. As an application, we look at a family of integral operators (e.g. the Fourier transform) as the action of continuous linear forms, and using the duality established between certain spaces, we obtain pointwise estimates that will allow us to give another proof of well-known bounds for the Fourier transform of $H^{p}$ functions (see [4], [12]). We can also improve the Féjer-Riesz inequality for harmonic extensions (see [5]) and we find a generalization considering Hardy spaces defined in terms of arbitrary kernels (see [14]). Our main tool will be given by the properties that the tent spaces satisfy (see [2], [1], [10]), and in particular their relation with a class of Carleson measures, for which we find a suitable atomic decomposition. We begin by giving some basic definitions.

Definition 1. Let $\boldsymbol{\Omega}=\left\{\boldsymbol{\Omega}_{x}\right\}_{x \in \mathbf{R}^{\mathbf{n}}}$ be a collection of measurable subsets, where $\Omega_{x} \subset \mathbf{R}_{+}^{\mathbf{n}+1}$. For a measurable function $f$ in $\mathbf{R}_{+}^{\mathbf{n}+1}$ we 
define the maximal function of $f$ with respect to $\Omega$ as

$$
A_{\Omega}^{\infty}(f)(x)=\sup _{(y, t) \in \Omega_{x}}|f(y, t)| .
$$

We will always assume that $\Omega$ is chosen so that $A_{\Omega}^{\infty}(f)$ is a measurable function. We also define

$$
T_{\Omega}^{p}=T_{\infty, \Omega}^{p}=\left\{f: A_{\Omega}^{\infty}(f) \in L^{p}\left(\mathbf{R}^{\mathbf{n}}\right)\right\},
$$

with $\|f\|_{T_{\Omega}^{p}}=\left\|A_{\Omega}^{\infty}(f)\right\|_{L^{p}\left(\mathbf{R}^{\mathrm{n}}\right)}$.

REMARK 2. It is clear that if $\Omega_{x}=\Gamma(x)$ then $T_{\Omega}^{p}$ is precisely the tent space $T_{\infty}^{p}$ of [2]. If $\Omega_{x}=\{(x, t): t>0\}$ then $A_{\Omega}^{\infty}(f)$ is the radial maximal function of $f$.

Definition 3. Suppose $\Omega=\left\{\Omega_{x}\right\}_{x \in \mathbf{R}^{\mathbf{n}}}$ is as above and $F$ is any subset of $\mathbf{R}^{\mathbf{n}}$. We define the tent over $F$, with respect to $\Omega$, as

$$
\widehat{F_{\Omega}}=\mathbf{R}_{+}^{\mathbf{n}+1} \backslash \bigcup_{x \notin F} \Omega_{x}
$$

We also set $\Omega_{x}(t)=\left\{y \in \mathbf{R}^{\mathbf{n}}:(y, t) \in \Omega_{x}\right\}$.

For a measure $\mu$ in $\mathbf{R}_{+}^{\mathbf{n}+1}$ we say that $\mu$ is an $(\Omega, \beta)$-Carleson measure $(\beta \geq 1)$ and write $\mu \in V_{\Omega}^{\beta}$ if

$$
\|\mu\|_{V_{\Omega}^{\beta}}=\sup _{Q} \frac{|\mu|\left(\widehat{Q_{\Omega}}\right)}{|Q|^{\beta}}<\infty,
$$

where the supremum is taken over all cubes $Q \subset \mathbf{R}^{\mathbf{n}}$.

REMARK 4. If $\Omega_{x}=\Gamma(x)$ then $\widehat{F_{\Omega}}=\widehat{F}$, the usual tent over $F$. If we choose $\Omega_{x}=\{(x, t): t>0\}$ then $\widehat{F_{\Omega}}=F \times \mathbf{R}^{+}$and it is denoted by $C(F)$.

Lemma 5. Suppose $F \subset \mathbf{R}^{\mathbf{n}}$ and $\Omega=\left\{\Omega_{x}\right\}_{x \in \mathbf{R}^{n}}$ are as above. Then

(i) $A_{\Omega}^{\infty}\left(\chi_{\widehat{F}_{\Omega}}\right)(x) \leq \chi_{F}(x)$ for all $x \in \mathbf{R}^{\mathbf{n}}$.

(ii) $A_{\Omega}^{\infty}\left(\chi_{F_{\Omega}}\right)(x)=\chi_{F}(x)$ if and only if $\Omega_{x} \cap \widehat{F_{\Omega}} \neq \varnothing$ for all $x \in F$.

(iii) If $\Omega$ is a symmetric family (that is, if $x \in \Omega_{y}(t)$ then $y \in$ $\left.\Omega_{x}(t)\right)$, we have that

$$
\widehat{F_{\Omega}}=\left\{(y, t) \in \mathbf{R}_{+}^{\mathbf{n}+1}: \Omega_{y}(t) \subset F\right\} .
$$

In particular if $\Omega_{x}=x+\Omega$, for a fixed $\Omega \subset \mathbf{R}_{+}^{\mathbf{n}+1}$, the symmetric condition holds if and only if $\Omega(t)=-\Omega(t)$, for all $t>0$. 
Proof. (i) Observe that

$$
\chi_{\widehat{F}_{\Omega}}(y, t)= \begin{cases}1, & \text { if }(y, t) \notin \Omega_{z}, \text { for all } z \notin F, \\ 0, & \text { otherwise. }\end{cases}
$$

Suppose $x \notin F$. Then if $(y, t) \in \Omega_{x}$ we have that $\chi_{\widehat{F}_{\Omega}}(y, t)=0$ (by (1)), and this shows (i).

(ii) $A_{\Omega}^{\infty}\left(\chi_{\widehat{F}_{\Omega}}\right)(x)=\chi_{F}(x)$ if and only if for all $x \in F, A_{\Omega}^{\infty}\left(\chi_{\widehat{F}_{\Omega}}\right)(x)$ $=1$ if and only if there exists $(y, t) \in \Omega_{x}$ such that $(y, t) \in \widehat{F_{\Omega}}$ if and only if $\Omega_{x} \cap \widehat{F_{\Omega}} \neq \varnothing$.

(iii) That $(y, t) \in \widehat{F_{\Omega}}$ means that $y \notin \Omega_{x}(t)$, for all $x \notin F$, which, by symmetry, is equivalent to saying that for all $x \notin F, x \notin \Omega_{y}(t)$; that is, $\Omega_{y}(t) \subset F$.

A simple example of a symmetric family of sets of the form $x+\Omega$ can be found in the comments previous to Lemma 11. Another example, for a general family of sets $\left\{\Omega_{x}\right\}$, is given by defining $\Omega_{n}(t)=(-n,-n+1)$, if $n \in \mathbf{Z}$, and $\Omega_{x}(t)=(-n-1,-n+1)$, if $n<x<n+1$.

Definition 6. We say that a measurable function $a: \mathbf{R}_{+}^{\mathbf{n}+1} \rightarrow \mathbf{C}$ is an $(\Omega, p)$-atom if there exists a cube $Q \subset \mathbf{R}^{\mathbf{n}}$ such that supp $a \subset \widehat{Q_{\Omega}}$, and $\|a\|_{\infty} \leq|Q|^{-1 / p}$.

We now give the proof of the atomic decomposition for the tent space $T_{\Omega}^{p}$. We restrict ourselves to the case $n=1$, but a similar proof also works in any other dimension. A related result is given in [6].

THEOREM 7. If $\Omega=\left\{\boldsymbol{\Omega}_{x}\right\}_{x \in \mathbf{R}}$ is a symmetric family of sets (as in Lemma 5-(iii)), such that $\Omega_{x}(t)$ is an interval, for all $(x, t) \in \mathbf{R}_{+}^{2}$, then, for $0<p \leq 1, f \in T_{\Omega}^{p}$ if and only if

$$
f \equiv \sum_{j} \lambda_{j} a_{j}
$$

where $a_{j}$ is an $(\Omega, p)$-atom and $\sum_{j}\left|\lambda_{j}\right|^{p}<\infty$. Moreover,

$$
\|f\|_{T_{\Omega}^{p}} \approx \inf \left\{\left(\sum_{j}\left|\lambda_{j}\right|^{p}\right)^{1 / p}\right\},
$$

where the infimum is taken over all sequences satisfying (2).

Proof. We first show the easy part, for which we will not make use of the extra hypotheses on $\Omega$. The only thing to observe is that $\|\cdot\|_{T_{\Omega}^{p}}$ 
is always a $p$-norm, for $0<p \leq 1$ and hence, if $f \equiv \sum_{j} \lambda_{j} a_{j}$, then $\|f\|_{T_{\Omega}^{p}}^{p} \leq \sum_{j}\left|\lambda_{j}\right|^{p}\left\|a_{j}\right\|_{T_{\Omega}^{p}}^{p}$. But, by (i) of the previous lemma:

$$
\begin{aligned}
\left\|a_{j}\right\|_{T_{\Omega}^{p}}^{p} & =\int_{\mathbf{R}}\left(A_{\Omega}^{\infty}\left(a_{j}\right)(x)\right)^{p} d x \\
& \leq \int_{\mathbf{R}}\left\|a_{j}\right\|_{\infty}^{p}\left(A_{\Omega}^{\infty}\left(\chi_{\widehat{Q_{j, \Omega}}}\right)(x)\right)^{p} d x \leq\left\|a_{j}\right\|_{\infty}^{p} \int_{\mathbf{R}} \chi_{Q_{j}}(x) d x \leq 1,
\end{aligned}
$$

and hence, $\|f\|_{T_{\Omega}^{p}}^{p} \leq \sum_{j}\left|\lambda_{j}\right|^{p}$.

For the converse we need the following observation: if $f \in T_{\Omega}^{p}$ and $\lambda>0$ then $\left\{x \in \mathbf{R}: A_{\Omega}^{\infty}(f)(x)>\lambda\right\}$ is an open set. In fact, if $A_{\Omega}^{\infty}(f)(x)>\lambda$, then there exists a point $(z, t) \in \Omega_{x}$ so that $|f(z, t)|>\lambda$. By hypotheses, we conclude that $x \in \Omega_{z}(t)$ and there exists an $\varepsilon>0$ such that if $|x-y|<\varepsilon$ then $y \in \Omega_{z}(t)$. Again, by symmetry, $(z, t) \in \Omega_{y}$ and so $A_{\Omega}^{\infty}(f)(y)>\lambda$ if $|x-y|<\varepsilon$. Set now $M_{k}=\left\{x \in \mathbf{R}: A_{\Omega}^{\infty}(f)(x)>2^{k}\right\}$, and write $M_{k}=\bigcup_{j \in \mathbf{Z}} I_{j}^{k}$, where $I_{j}^{k}$ is an open interval and $I_{j}^{k} \cap I_{j^{\prime}}^{k}=\varnothing$ if $j \neq j^{\prime}$. Since $f \in T_{\Omega}^{p}, I_{j}^{k}$ is bounded for all $j, k \in \mathbf{Z}$. Set

$$
a_{j, k} \equiv \lambda_{j, k}^{-1} f\left(\chi_{\widehat{I_{j, \Omega}^{k}}}-\sum_{I_{l}^{k+1} \subset I_{j}^{k}} \chi_{\widehat{I_{l, \Omega}^{k+1}}}\right),
$$

where $\lambda_{j, k}=2^{k+1}\left|I_{j}^{k}\right|^{1 / p}$. It is clear that $\operatorname{supp} a_{j, k} \subset \widehat{I_{j, \Omega}^{k}}$ and

$$
\sum_{j, k}\left|\lambda_{j, k}\right|^{p}=\sum_{k} 2^{p(k+1)}\left|M_{k}\right| \leq C\|f\|_{T_{\Omega}^{p}}^{p}<\infty,
$$

and so it remains to show that $f \equiv \sum_{j, k} \lambda_{j, k} a_{j, k}$ and $\left\|a_{j, k}\right\|_{\infty} \leq$ $\left|I_{j}^{k}\right|^{-1 / p}$. Let $(x, t) \in \widehat{I_{j, \Omega}^{k}}$ and suppose $|f(x, t)|>2^{k+1}$. Let $y \in$ $\Omega_{x}(t)$. Then $(x, t) \in \Omega_{y}$ and hence $y \in M_{k+1}$. Therefore $\Omega_{x}(t) \subset$ $M_{k+1}$ and there exists a unique $l \in \mathbf{Z}$ so that $\Omega_{x}(t) \subset I_{l}^{k+1}$. Since $\Omega_{x}(t) \subset I_{j}^{k}$ then $I_{l}^{k+1} \subset I_{j}^{k}$. But if $I_{l^{\prime}}^{k+1} \subset I_{j}^{k}$ and $l \neq l^{\prime}$ then $\widehat{I_{l, \Omega}^{k+1}} \cap \widehat{I_{l^{\prime}, \Omega}^{k+1}} \neq \varnothing$. In fact, if $(z, s) \in \widehat{I_{l, \Omega}^{k+1}} \cap \widehat{I_{l^{\prime}, \Omega}^{k+1}}$ then $\Omega_{z}(s) \subset$ $I_{l}^{k+1} \cap I_{l^{\prime}}^{k+1}$, which is a contradiction. Thus,

$$
\chi_{\widehat{I_{J, \Omega}^{k}}}(x, t)-\sum_{I_{r}^{k+1} \subset I_{J}^{k}} \chi_{\widehat{I_{r, \Omega}^{k+1}}}(x, t)=0 .
$$

Therefore, for all $(x, t) \in \widehat{I_{j, \Omega}^{k}}$,

$$
\left|a_{j, k}(x, t)\right| \leq 2^{-(k+1)}\left|I_{j}^{k}\right|^{-1 / p} 2^{k+1}=\left|I_{j}^{k}\right|^{-1 / p} .
$$


Finally, if $(x, t) \in \mathbf{R}_{+}^{2}$ and $2^{l}<|f(x, t)| \leq 2^{l+1}$ then $\Omega_{x}(t) \subset M_{l}$. Let $K \in \mathbf{Z}$ be the greatest integer satisfying $\Omega_{x}(t) \subset M_{K}$ (it is clear that we can find such a number since $A_{\Omega}^{\infty}(f)(x)<\infty$, a.e. $\left.x \in \mathbf{R}\right)$. Let $s \in \mathbf{Z}$ so that $\Omega_{x}(t) \subset I_{s}^{K}$. We want to show that if

$$
g_{j, k}(x, t)=\chi_{I_{J, \Omega}^{k}}(x, t)-\sum_{I_{r}^{k+1} \subset I_{j}^{k}} \chi_{\widehat{I_{r, \Omega}^{k+1}}}(x, t),
$$

then $\sum_{j, k} g_{j, k}(x, t)=1$. If $\Omega_{x}(t) \subset I_{j}^{k}$ then $k \leq K$. Suppose that $k<K$ and $(x, t) \in \widehat{I_{j, \Omega}^{k}}$, then $I_{s}^{K} \subset I_{r}^{k+1} \subset I_{j}^{k}$ for some $r \in \mathbf{Z}$ and hence $g_{j, k}(x, t)=0$. If $(x, t) \in \widehat{I_{j, \Omega}^{K}}$ then clearly $j=s$ and $g_{K, s}(x, t)=1$.

We observe that in the previous proof, we obtained the atomic decomposition for all $0<p<\infty$. An immediate application of this theorem is given by the following duality result. We first recall that for the case when $\Omega_{x}$ is the cone $\Gamma(x)$, it was proved in [2] and [1] that the space of Carleson measures of order $1 / p(0<p \leq 1)$ could be identified as the dual of the tent space $T_{\infty}^{p}$ (see Theorem 16). For the general case we are considering, we restrict our study only to the inclusion needed in order to obtain the estimates we mention below.

THEOREM 8. Suppose $\Omega$ is a family of sets satisfying the hypotheses of the previous theorem and $0<p \leq 1$. Then, for all $f \in T_{\Omega}^{p}$ and $\mu \in V_{\Omega}^{1 / p}$,

$$
\left|\int_{\mathbf{R}_{+}^{2}} f(x, t) d \mu(x, t)\right| \leq\|f\|_{T_{\Omega}^{p}}\|\mu\|_{V_{\Omega}^{1 / p}} .
$$

That is, $V_{\Omega}^{1 / p} \hookrightarrow\left(T_{\Omega}^{p}\right)^{*}$.

Proof. Let $f \in T_{\Omega}^{p}$ and $\mu \in V_{\Omega}^{1 / p}$, and write $f \equiv \sum_{j} \lambda_{j} a_{j}$, as in Theorem 7. Then,

$$
\begin{aligned}
& \left|\int_{\mathbf{R}_{+}^{2}} f(x, t) d \mu(x, t)\right| \leq \sum_{j}\left|\lambda_{j}\right| \int_{\widehat{I_{j, \Omega}}}\left|a_{j}(x, t)\right| d|\mu|(x, t) \\
& \quad \leq \sum_{j}\left|\lambda_{j}\right|\left\|a_{j}\right\|_{\infty}|\mu|\left(\widehat{I_{j, \Omega}}\right) \leq \sum_{j}\left|\lambda_{j}\right|\left|I_{j}\right|^{-1 / p}\|\mu\|_{V_{\Omega}^{1 / p}\left|I_{j}\right|^{1 / p}} \\
& \quad \leq\left(\sum_{j}\left|\lambda_{j}\right|^{p}\right)^{1 / p}\|\mu\|_{V_{\Omega}^{1 / p}} .
\end{aligned}
$$


REMARK 9. (i) In the proof of the previous theorem, if $p=1$, we can give a direct argument without using the atomic decomposition. In fact, if $f \in T_{\Omega}^{1}$ and if we consider the set $F^{\lambda}=\left\{y \in \mathbf{R}: A_{\Omega}^{\infty}(f)(y)>\right.$ $\lambda\}$, then

$$
\left\{(x, t) \in \mathbf{R}_{+}^{2}:|f(x, t)|>\lambda\right\} \subset \widehat{F_{\Omega}^{\lambda}} .
$$

In fact, if $|f(x, t)|>\lambda, A_{\Omega}^{\infty}(f)(z) \leq \lambda$, implies that $(x, t) \notin \Omega_{z}$ and, hence,

$$
(x, t) \in \mathbf{R}_{+}^{2} \backslash\left(\bigcup_{z \notin F^{\lambda}} \Omega_{z}\right)=\widehat{F_{\Omega}^{\lambda}} .
$$

As we saw before, $F^{\lambda}$ is an open set and hence $F^{\lambda}=\bigcup_{j} I_{j}$. Moreover, by symmetry, $\widehat{F_{\Omega}^{\lambda}} \subset \bigcup_{j} \widehat{I_{j, \Omega}}$, and hence, for $\mu \in V_{\Omega}^{1}$, we have

$$
\begin{aligned}
& \left|\int_{\mathbf{R}_{+}^{2}} f(x, t) d \mu(x, t)\right| \\
& \quad \leq \int_{0}^{\infty}|\mu|\left(\left\{(x, t) \in \mathbf{R}_{+}^{2}:|f(x, t)|>\lambda\right\}\right) d \lambda \\
& \quad \leq \int_{0}^{\infty}|\mu|\left(\widehat{F_{\Omega}^{\lambda}}\right) d \lambda \leq \sum_{j} \int_{0}^{\infty}|\mu|\left(\widehat{I_{j, \Omega}}\right) d \lambda \\
& \quad \leq\|\mu\|_{V_{\Omega}^{1}} \int_{0}^{\infty}\left|\bigcup_{j} I_{j}\right| d \lambda=\|\mu\|_{V_{\Omega}^{1}}\|f\|_{T_{\Omega}^{1}}
\end{aligned}
$$

(ii) If $\Omega$ satisfies that for every compact $K \subset \mathbf{R}_{+}^{2}$, the set $\{x \in \mathbf{R}$ : $\left.\Omega_{x} \cap K \neq \varnothing\right\}$ has finite measure, then using the ideas of [2], it is easy to show that in fact equality holds; namely $V_{\Omega}^{1 / p}=\left(T_{\Omega}^{p}\right)^{*}$. We do not know what happens in the general case.

As was proved in [4] the non-tangential maximal function and the radial maximal function of Poisson integrals of functions (distributions) in the Hardy space $H^{p}\left(\mathbf{R}^{\mathbf{n}}\right)$ have an equivalent $L^{p}$ " "norm", $p>0$. This leads us to consider how this result could be extended for all functions in the tent spaces $T_{\infty}^{p}$ relative to both cones $\Gamma(x)$ and lines $\{(x, t): t>0\}$. From the point of view of the dual spaces we see that the latter is a much bigger space than the former. We give the details in what follows.

EXAMPLE 10. If $\Omega_{x}=\{(x, t): t>0\}$ then $\widehat{O_{\Omega}}=C(O)=O \times \mathbf{R}^{+}$. Let us denote $V_{\text {rad }}^{\alpha}=V_{\Omega}^{\alpha}$, where $\Omega_{x}$ is the vertical line above $x$. 
First suppose that $0<\alpha \leq 1, f \in L^{1 /(1-\alpha)}\left(\mathbf{R}^{\mathbf{n}}\right)$ and $\sigma$ is a positive finite measure in $\mathbf{R}^{+}$. Then

$$
d \mu(x, t)=f(x) d x d \sigma(t) \in V_{\mathrm{rad}}^{\alpha} .
$$

In fact, if $O \subset \mathbf{R}^{\mathbf{n}}$ then

$$
\begin{aligned}
\left|\int_{C(O)} d \mu(x, t)\right| & \leq\left(\int_{O}|f(x, t)| d x\right)\left(\int_{0}^{\infty} d \sigma(t)\right) \\
& \leq\|\sigma\|\|f\|_{L^{1 /(1-\alpha)}|O|^{\alpha}}
\end{aligned}
$$

An example of a measure that is in $V^{\alpha}$ but not in $V_{\text {rad }}^{\alpha}$ is the Dirac mass at the point $\left(x_{0}, t_{0}\right) \in \mathbf{R}_{+}^{\mathbf{n}+1}$. This follows by considering a collection of cubes converging to $x_{0}$.

However, for the case $\alpha>1$ we get that

$$
V_{\mathrm{rad}}^{\alpha}=\{0\} \text {. }
$$

To show this fix a cube $Q \subset \mathbf{R}^{\mathbf{n}}$ and $N \in \mathbf{Z}^{+}$. Decompose $Q$ in $2^{n N}$ subcubes $Q_{i}$ such that $\dot{Q}_{i} \cap \dot{Q}_{j}=\varnothing, i \neq j, Q=\bigcup_{i} Q_{i}$ and $\left|Q_{i}\right|=|Q| / 2^{n N}$. Now, if $\mu \in V_{\text {rad }}^{\alpha}$ we have

$$
\begin{aligned}
|\mu|(C(Q)) & \leq|\mu|\left(\bigcup_{i} C\left(Q_{i}\right)\right) \leq \sum_{i}|\mu|\left(C\left(Q_{i}\right)\right) \leq C_{\mu} \sum_{i}\left|Q_{i}\right|^{\alpha} \\
& =C_{\mu} \sum_{i=1}^{2^{n N}} \frac{|Q|^{\alpha}}{2^{\alpha n N}}=C_{\mu}|Q|^{\alpha} 2^{n N(1-\alpha)} \rightarrow 0 \text { as } N \rightarrow \infty .
\end{aligned}
$$

Hence $\mu \equiv 0$.

Our first application of the duality result, deals with pointwise estimates for the Fourier transform of functions satisfying an $H^{p}$-type condition. Consider an increasing function $\psi: \mathbf{R}^{+} \rightarrow \mathbf{R}^{+}, \psi$ a $C^{1}$ change of variables. Define the sets $\Omega_{x}=\left\{(y, t) \in \mathbf{R}_{+}^{2}:|x-y|<\right.$ $\psi(t)\}$. It is clear that $\Omega_{x}$ satisfies the hypotheses of Theorem 7. Observe that

$$
\widehat{I_{\Omega}}=\left\{(y, t) \in \mathbf{R}_{+}^{2}: d(y, \mathbf{R} \backslash I) \geq \psi(t)\right\} .
$$

We say that a function $f$ belongs to $H_{\Omega}^{p}$ if $P I(f)(x, t)=P_{t} * f(x)$ belongs to the space $T_{\Omega}^{p}$, where $P$ is the Poisson kernel in $\mathbf{R}$.

LEMMA 11. Let $\psi$ and $\Omega$ be as before, and suppose $0<p<1$. Consider the function $\varphi(t)=\psi^{1 / p-2}(t) \psi^{\prime}(t)$. Then, if $g \in L^{\infty}$ and 
$d \mu(y, t)=g(y) \varphi(t) d y d t$, we have that $\mu \in V_{\Omega}^{1 / p}$ and $\|\mu\|_{V_{\Omega}^{1 / p}} \leq$ $C_{p}\|g\|_{\infty}$.

Proof. Let $I=(a, b)$. Then, by (4):

$$
\begin{aligned}
|\mu|\left(\widehat{I_{\Omega}}\right) \leq & \int_{a}^{b} \int_{0}^{\psi^{-1}(d(y, \mathbf{R} \backslash I))}|g(y)| \varphi(t) d t d y \\
\leq & \|g\|_{\infty}\left(\int_{a}^{(a+b) / 2} \int_{0}^{\psi^{-1}(y-a)} \psi^{1 / p-2}(t) \psi^{\prime}(t) d t d y\right. \\
& \left.\quad+\int_{(a+b) / 2}^{b} \int_{0}^{\psi^{-1}(b-y)} \psi^{1 / p-2}(t) \psi^{\prime}(t) d t d y\right) .
\end{aligned}
$$

But,

and hence,

$$
\int_{0}^{\psi^{-1}(r)} \psi^{1 / p-2}(t) \psi^{\prime}(t) d t=\frac{p}{1-p} r^{1 / p-1}
$$

$$
\begin{aligned}
|\mu|\left(\widehat{I_{\Omega}}\right) \leq C_{p}\|g\|_{\infty}\left(\int_{a}^{(a+b) / 2}(y-a)^{1 / p-1} d y\right. \\
\left.+\int_{(a+b) / 2}^{b}(b-y)^{1 / p-1} d y\right) \\
\leq C_{p}\|g\|_{\infty}(b-a)^{1 / p} .
\end{aligned}
$$

Proposition 12. Suppose $\psi, \Omega, \varphi$ and $0<p<1$ are as in the previous lemma. Then, for $f \in H_{\Omega}^{p}$,

$$
|\hat{f}(x)| \leq C_{p}\|f\|_{H_{\Omega}^{p}}\left(\int_{0}^{\infty} e^{-2 \pi|x| t} \varphi(t) d t\right)^{-1} .
$$

Proof. Fix $0<\varepsilon<1$ and set $\varphi_{\varepsilon}(t)=\varphi(t) \chi_{(\varepsilon, 1 / \varepsilon)}(t)$. If we define $d \mu_{\varepsilon}(y, t)=e^{-i x y} \varphi_{\varepsilon}(t) d y d t$, by Lemma 11 , we have that $\left\|\mu_{\varepsilon}\right\|_{V_{\Omega}^{1 / p}} \leq$ $C_{p}$. Now, if $f \in H_{\Omega}^{p}$ then $P_{t} * f \in T_{\Omega}^{p}$, and by Theorem 8 ,

$$
\left|\int_{\mathbf{R}_{+}^{2}} P_{t} * f(y) d \mu_{\varepsilon}(y, t)\right| \leq C_{p}\|f\|_{H_{\Omega}^{p}}
$$

But,

$$
\left|\int_{\mathbf{R}_{+}^{2}} P_{t} * f(y) d \mu_{\varepsilon}(y, t)\right|=|\hat{f}(x)| \int_{\varepsilon}^{1 / \varepsilon} e^{-2 \pi|x| t} \varphi(t) d t
$$


EXAMPLE 13. (i) If $\psi(t)=t$ in the previous result, we get the classical estimate for the Fourier transform of functions in $H^{p}$ :

$$
|\hat{f}(x)| \leq C_{p}|x|^{1 / p-1} \text {. }
$$

We will give more details about this result in Corollary 20.

(ii) If for example $\psi(t)=e^{t}-1$, so that $\Omega_{x}$ is a domain containing the cone $\Gamma(x)$, then $\varphi(t)=\left(e^{t}-1\right)^{1 / p-2} e^{t}$, and the integral $\int_{0}^{\infty} e^{-2 \pi|x| t} \varphi(t) d t$ converges if and only if $|x|>(1-p) /(2 \pi p)$. Hence, $\hat{f}(x)=0$ if $|x| \leq(1-p) /(2 \pi p)$ and $f \in H_{\Omega}^{p}$. Therefore, since $f_{r}(x)=f(r x) \in H_{\Omega}^{p}$, if $f \in H_{\Omega}^{p}$, one finds that $\hat{f}(x)=0$, for all $x \in \mathbf{R}$, and so $H_{\Omega}^{p}=0$.

(iii) The above calculations show that, in fact, a necessary condition for $H_{\Omega}^{p}$ to be nontrivial is that the Laplace transform of $\varphi$,

$$
\mathscr{L} \varphi(x)=\int_{0}^{\infty} e^{-x t} \varphi(t) d t<\infty,
$$

for all $x \neq 0$, which, for example, happens if for all $s>0$, there exists a constant $C_{s}>0$ such that $\psi(t) \leq C_{s} e^{s t}$, for all $t>0$.

We give now a characterization of the class of Carleson measures in terms of the boundedness of the mean operator. Some related questions can be found in [7] and [9]. Given a symmetric family $\Omega$ such that $\Omega_{x}(t)$ is an open interval and for all intervals $I \subset \mathbf{R}$ there exists $(x, t) \in \mathbf{R}_{+}^{2}$ with $\Omega_{x}(t)=I$ (these conditions hold if, for example, $\Omega$ is given by a function $\psi$ as in Lemma 11), we define the following mean operator:

$$
T_{\Omega} f(x, t)=\frac{1}{\left|\Omega_{x}(t)\right|} \int_{\Omega_{x}(t)} f(y) d y .
$$

We extend the notion of Carleson measure to consider the case of weights simply by saying that the pair $(\mu, u) \in V_{\Omega}^{\alpha}$ if

$$
|\mu|\left(\widehat{I_{\Omega}}\right) \leq C(u(I))^{\alpha},
$$

where $u$ is a positive and locally integrable function in $\mathbf{R}$ and $u(I)=$ $\int_{I} u(x) d x$. Thus, in our previous notation, $\mu \in V_{\Omega}^{\alpha}$ means that $(\mu, 1) \in V_{\Omega}^{\alpha}$. Recall that $A_{p}$ denotes the class of Muckenhoupt's weights (see [5]).

THeOREM 14. (i) If $\alpha \geq 1, p>0$ and $T_{\Omega}: L^{p}(\mathbf{R}, u) \rightarrow L^{\alpha p}\left(\mathbf{R}_{+}^{2}, d \mu\right)$ is a bounded operator, then $(\mu, u) \in V_{\Omega}^{\alpha}$, and $\|\mu\| \leq\left\|T_{\Omega}\right\|^{\alpha p}$, where $\|\mu\|$ is the best constant in (5). 
(ii) If $u \in A_{p}, p>1$ and $(\mu, u) \in V_{\Omega}^{\alpha}, \alpha \geq 1$, then $T_{\Omega}: L^{p}(\mathbf{R}, u)$ $\rightarrow L^{\alpha p}\left(\mathbf{R}_{+}^{2}, d \mu\right)$ is a bounded operator, and $\left\|T_{\Omega}\right\| \leq C\|\mu\|^{1 /(\alpha p)}$.

(iii) Fix $1<p<\infty$. Then, $\mu \in V_{\Omega}^{\alpha}$ if and only if $T_{\Omega}: L^{p}(\mathbf{R}) \rightarrow$ $L^{\alpha p}\left(\mathbf{R}_{+}^{2}, d \mu\right)$ is a bounded operator.

(iv) Let $\delta_{\left(x_{0}, t_{0}\right)}$ denote the Dirac delta at $\left(x_{0}, t_{0}\right) \in \mathbf{R}_{+}^{2}$. Then the operator $T_{\Omega}: L^{p}(\mathbf{R}, u) \rightarrow L^{p}\left(\mathbf{R}_{+}^{2}, \delta_{\left(x_{0}, t_{0}\right)}\right)$ is bounded, for all $\left(x_{0}, t_{0}\right)$ $\in \mathbf{R}_{+}^{2}$, and $\left\|T_{\Omega}\right\| \leq C_{p}\left(u\left(\Omega_{x_{0}}\left(t_{0}\right)\right)\right)^{-1 / p}$, if and only if $u \in A_{p}$.

Proof. (i) Evaluate $T_{\Omega} f$, if $f=\chi_{I}$, to get

$$
T_{\Omega} \chi_{I}(x, t)=\frac{\left|\Omega_{x}(t) \cap I\right|}{\left|\Omega_{x}(t)\right|} \geq \chi_{\widehat{I}_{\Omega}}(x, t),
$$

and hence,

$$
\mu\left(\widehat{I_{\Omega}}\right)^{1 /(\alpha p)} \leq\left\|T_{\Omega} \chi_{I}\right\|_{L^{\alpha p}(d \mu)} \leq\left\|T_{\Omega}\right\|\left\|\chi_{I}\right\|_{L^{p}(u)}=\left\|T_{\Omega}\right\| u(I)^{1 / p} .
$$

(ii) As we saw in Remark 9, if $F^{t}=\left\{y \in \mathbf{R}: A_{\Omega}^{\infty}\left(T_{\Omega} f\right)(y)>t\right\}$, then

$$
\left\{(x, s) \in \mathbf{R}_{+}^{2}: T_{\Omega} f(x, s)>t\right\} \subset \widehat{F_{\Omega}^{t}} .
$$

If $M$ denotes the Hardy-Littlewood maximal function, it is clear that by symmetry, $A_{\Omega}^{\infty} f(y) \leq M f(y)$, and hence,

$$
\begin{aligned}
\mu(\{(x, s) & \left.\left.\in \mathbf{R}_{+}^{2}: T_{\Omega} f(x, s)>t\right\}\right) \leq \mu\left(\widehat{F_{\Omega}^{t}}\right) \\
& \leq\|\mu\|\left(u\left(F^{t}\right)\right)^{\alpha} \leq\|\mu\|(u(\{M f>t\}))^{\alpha} .
\end{aligned}
$$

Using now that $L^{p}(u) \subset L^{p, \alpha p}(u)$, the classical Lorentz space,

$$
\begin{aligned}
\left\|T_{\Omega} f\right\|_{L^{\alpha p}(d \mu)} & \leq C\left(\int_{0}^{\infty} t^{\alpha p-1} \mu\left(\left\{(x, s) \in \mathbf{R}_{+}^{2}: T_{\Omega} f(x, s)>t\right\}\right) d t\right)^{1 /(\alpha p)} \\
& \leq C\|\mu\|^{1 /(\alpha p)}\left(\int_{0}^{\infty} t^{\alpha p-1}(u(\{M f>t\}))^{\alpha} d t\right)^{1 /(\alpha p)} \\
& =C\|\mu\|^{1 /(\alpha p)}\|M f\|_{L^{p, \alpha p}(u)} \leq C\|\mu\|^{1 /(\alpha p)}\|f\|_{L^{p}(u)} .
\end{aligned}
$$

(iii) It is a trivial consequence of (i) and (ii).

(iv) We first observe that for all $u \in L_{\text {loc }}^{1},(\delta, u) \in V_{\Omega}^{\alpha}$, and $\|\delta\| \leq$ $\left(u\left(\Omega_{x_{0}}\left(t_{0}\right)\right)\right)^{-\alpha}$. Hence, if $u \in A_{p}$, we get the boundedness of $T_{\Omega}$, by (ii). Conversely, if $f \in L^{p}(u)$,

$$
\begin{aligned}
\left\|T_{\Omega} f\right\|_{L^{p}(\delta)} & =\frac{1}{\left|\Omega_{x_{0}}\left(t_{0}\right)\right|} \int_{\Omega_{x_{0}}\left(t_{0}\right)} u^{-1}(x) f(x) u(x) d x \\
& \leq C\left(u\left(\Omega_{x_{0}}\left(t_{0}\right)\right)\right)^{-1 / p}\|f\|_{L^{p}(u)} .
\end{aligned}
$$


Taking the supremum when $\|f\|_{L^{p}(u)} \leq 1$,

$$
\frac{1}{\left|\Omega_{x_{0}}\left(t_{0}\right)\right|}\left(\int_{\Omega_{x_{0}}\left(t_{0}\right)} u^{-p^{\prime}+1}(x) d x\right)^{1 / p^{\prime}} \leq\left(\int_{\Omega_{x_{0}}\left(t_{0}\right)} u(x) d x\right)^{-1 / p} .
$$

Hence,

$$
\left(\frac{1}{\left|\Omega_{x_{0}}\left(t_{0}\right)\right|} \int_{\Omega_{x_{0}}\left(t_{0}\right)} u(x) d x\right)\left(\frac{1}{\left|\Omega_{x_{0}}\left(t_{0}\right)\right|} \int_{\Omega_{x_{0}}\left(t_{0}\right)} u^{-p^{\prime}+1}(x) d x\right)^{p-1} \leq C,
$$

and by the hypotheses on $\Omega$, this implies $u \in A_{p}$.

We consider now the usual case when $\Omega_{x}$ is a cone, to obtain some results in the classical theory of Hardy spaces.

Definition 15. Suppose $\sigma$ is a Borel measure in $\mathbf{R}^{+}$. We say that $\sigma$ is a measure of order $\beta$, with $\beta \geq 0$, if there exists a constant $C>0$ such that

$$
\int_{0}^{t} d|\sigma| \leq C t^{\beta}, \quad \text { for all } t>0 .
$$

In this case, we write $\sigma \in M^{\beta}$ and also $\|\sigma\|_{M^{\beta}}=\inf \{C: C$ satisfies (6)\} .

The following result corresponds to Theorem 8 .

Theorem 16 (see [2], [1]). For $0<p \leq 1$, the pairing $(f, d \mu) \rightarrow$ $\int_{\mathbf{R}_{+}^{\mathrm{n}+1}} f(x, t) d \mu(x, t)$, with $f \in T_{\infty}^{p}$ and $\mu \in V^{1 / p}$, realizes the duality of $T_{\infty}^{p}$ with $V^{1 / p}$.

For our next result, we need to introduce a densely defined bilinear functional. We will restrict the action of this operator, when considering distributions in the Hardy space $H^{p}\left(\mathbf{R}^{\mathbf{n}}\right)$, to the dense subspace $\mathscr{S}_{0}$ of those functions in the class $\mathscr{S}$ with mean zero.

Definition 17. Fix $1 \leq q \leq \infty$. Suppose $F: \mathbf{R}^{\mathbf{n}} \times \mathbf{R}^{\mathbf{n}} \rightarrow \mathbf{C}$ is a measurable function such that if we set $F_{z}(x)=F(z, x), z, x \in \mathbf{R}^{\mathbf{n}}$; then $F_{z} \in L^{q}\left(\mathbf{R}^{\mathbf{n}}\right)$. Let $\alpha \geq 0$. For $g \in \mathscr{S}_{0}$, set

$$
R_{F}(g)(x, z)=\int_{\mathbf{R}^{\mathbf{n}}} g(y) F(z, y+x) d y .
$$

We define, for $\sigma \in M^{\alpha}$,

$$
T_{F}(g, \sigma)(z)=\int_{0}^{\infty}\left(R_{F}(g)(\cdot, z) * P_{t}\right)(0) d \sigma(t),
$$


where $P(x)=c_{n}\left(1+|x|^{2}\right)^{-(n+1) / 2}$ is the Poisson kernel in $\mathbf{R}^{\mathbf{n}}$, and $P_{t}(x)=t^{-n} P(x / t)$.

EXAMPLE 18. Suppose $q=\infty$ and $F(z, x)=e^{-i x z}$. Then $\|F\|_{\infty}$ $=1$ and if $g \in \mathscr{S}_{0}$ we have that

$$
R_{F}(g)(x, z)=\int_{\mathbf{R}^{\mathbf{n}}} g(y) e^{-i(x+y) z} d y=e^{-i x z} \hat{g}(z) .
$$

Hence,

$$
\left(R_{F}(g)(\cdot, z) * P_{t}\right)(0)=\int_{\mathbf{R}^{\mathbf{n}}} e^{-i x z} \hat{g}(z) P_{t}(x) d x=\hat{g}(z) \widehat{P}_{t}(z)
$$

If $0<p<1$ and we consider the measure $d \sigma(t)=t^{n(1 / p-1)-1} d t$, then we have that $\sigma \in M^{n(1 / p-1)}$, since

$$
\int_{0}^{t} d|\sigma|(t)=\frac{t^{n(1 / p-1)}}{n(1 / p-1)}
$$

and so,

$$
\|\sigma\|_{M^{n(1 / p-1)}}=\frac{1}{n(1 / p-1)}
$$

Therefore,

$$
\begin{aligned}
T_{F}(g, \sigma)(z) & =\int_{0}^{\infty} \hat{g}(z) \widehat{P}_{t}(z) t^{n(1 / p-1)-1} d t \\
& =c_{n} \hat{g}(z) \int_{0}^{\infty} e^{-2 \pi t|z|} t^{n(1 / p-1)-1} d t
\end{aligned}
$$

and the integral is finite since $n(1 / p-1)>0$.

Theorem 19. Suppose $1 \leq q \leq \infty, \alpha \geq n / q$ and $1 / p=\alpha / n+1 / q^{\prime}$, so that $0<p<1$. Then

$$
\left|T_{F}(g, \sigma)(z)\right| \leq c_{n}\|\sigma\|_{M^{\alpha}}\left\|F_{z}\right\|_{L^{q}\left(\mathbf{R}^{\mathbf{n}}\right)}\|g\|_{H^{p}\left(\mathbf{R}^{\mathbf{n}}\right)},
$$

for all $\sigma \in M^{\alpha}$ and $g \in \mathscr{S}_{0}$.

Proof. The proof is a consequence of the nontangential maximal characterization of $H^{p}\left(\mathbf{R}^{\mathbf{n}}\right)$ (see [4]): $\|g\|_{H^{p}\left(\mathbf{R}^{\mathbf{n}}\right)} \approx\|P I(g)\|_{T_{\infty}^{p}}$, where $P I(g)(x, t)=\left(P_{t} * g\right)(x)$. To estimate this quantity we use Theorem 
16, $\left(T_{\infty}^{p}\right)^{*}=V^{1 / p}, 0<p \leq 1$ :

$$
\begin{aligned}
T_{F}(g, \sigma)(z) & =\int_{0}^{\infty}\left(\int_{\mathbf{R}^{\mathbf{n}}} P_{t}(u) R_{F}(g)(u, z) d u\right) d \sigma(t) \\
& =\int_{\mathbf{R}_{+}^{\mathbf{n}+1}} g(y)\left(\int_{\mathbf{R}^{\mathbf{n}}} P_{t}(u) F(z, y+u) d u\right) d y d \sigma(t) \\
& =\int_{\mathbf{R}_{+}^{\mathbf{n}+1}} g(y)\left(\int_{\mathbf{R}^{\mathbf{n}}} P_{t}(v-y) F(z, v) d v\right) d y d \sigma(t) \\
& =\int_{\mathbf{R}_{+}^{\mathbf{n}+1}} P I(g)(v, t) F(z, v) d v d \sigma(t) .
\end{aligned}
$$

For a fixed $z$, consider the measure

$$
d \mu(v, t)=F_{z}(v) d v d \sigma(t) .
$$

Then, we claim that $\mu \in V^{1 / p}$ and $\|\mu\|_{V^{1 / p}} \leq\|\sigma\|_{M^{\alpha}}\left\|F_{z}\right\|_{L^{q}}$. Thus,

$$
\begin{aligned}
\left|T_{F}(g, \sigma)(z)\right| & \leq \int_{\mathbf{R}_{+}^{\mathrm{n}+1}}|P I(g)(v, t)| d|\mu|(v, t) \\
& \leq\|P I(g)\|_{T_{\infty}^{p}}\|\mu\|_{V^{1 / p}} \leq c_{n}\|\sigma\|_{M^{\alpha}}\left\|F_{z}\right\|_{L^{q}}\|g\|_{H^{p}} .
\end{aligned}
$$

To prove the claim, it suffices to show that if $f \in L^{q}\left(\mathbf{R}^{\mathbf{n}}\right), 1 \leq q \leq$ $\infty, \sigma \in M^{\alpha}$, with $\beta=1 / q^{\prime}+\alpha / n \geq 1$ and we set $d \mu(x, t)=$ $f(x) d x d \sigma(t)$, then $\mu \in V^{\beta}$ and $\|\mu\|_{V^{\beta}} \leq\|\sigma\|_{M^{\alpha}}\|f\|_{L^{q}}$. Now, for a cube $Q \subset \mathbf{R}^{\mathbf{n}}$,

$$
\begin{aligned}
& |\mu|(\widehat{Q}) \leq\left(\int_{Q}|f(x)| d x\right)\left(\int_{0}^{|Q|^{1 / n}} d|\sigma|(t)\right) \\
& \leq\|f\|_{L^{q}}|Q|^{1 / q^{\prime}}\|\sigma\|_{M^{\alpha}}|Q|^{\alpha / n}=\|f\|_{L^{q}}\|\sigma\|_{M^{\alpha}}|Q|^{\beta},
\end{aligned}
$$

and so, $\|\mu\|_{V^{\beta}} \leq\|f\|_{L^{q}}\|\sigma\|_{M^{\alpha}}$.

Corollary 20. If $0<p \leq 1$ and $g \in \mathscr{S}_{0}\left(\mathbf{R}^{\mathbf{n}}\right)$, then

$$
|\hat{g}(z)| \leq C_{n, p}|z|^{n(1 / p-1)}\|g\|_{H^{p}}
$$

for all $z \in \mathbf{R}^{\mathbf{n}}$.

Proof. It suffices to consider the case $0<p<1$ and $z \neq 0$. We recall that by Example 18 we have

$$
T_{F}(g, \sigma)(z)=c_{n} \hat{g}(z) \int_{0}^{\infty} e^{-2 \pi t|z|} t^{n(1 / p-1)-1} d t .
$$


But,

$$
\begin{aligned}
& \int_{0}^{\infty} e^{-2 \pi t|z|} t^{n(1 / p-1)-1} d t \\
& \quad=C|z|^{-n(1 / p-1)} \int_{0}^{\infty} e^{-2 \pi u} u^{n(1 / p-1)-1} d u=C_{n, p}|z|^{-n(1 / p-1)}
\end{aligned}
$$

Hence, by the theorem,

$$
\left|T_{F}(g, \sigma)(z)\right| \leq c_{n}\|\sigma\|_{M^{\alpha}}\left\|F_{z}\right\|_{\infty}\|g\|_{H^{p}\left(\mathbf{R}^{\mathrm{n}}\right)} ;
$$

that is,

$$
C_{n, p}|\hat{g}(z)||z|^{-n(1 / p-1)} \leq \frac{c_{n}}{n(1 / p-1)}\|g\|_{H^{p}}
$$

which gives the result.

REMARK 21. Corollary 20 was first proved in [4], using a different approach. Later in [12], it was also proved using the atomic characterization of $H^{p}$. We want to give yet another simple proof using now the duality of the $H^{p}$ spaces. In [3] it is shown that $\left(H^{p}\left(\mathbf{R}^{\mathbf{n}}\right)\right)^{*}=\dot{B}_{\infty}^{n(1 / p-1), \infty}, 0<p<1$, where the norm on this Besov space coincides with the Lipschitz norm of order $n(1 / p-1)$ (see [11]); namely,

$$
\|f\|_{\dot{B}_{\infty}^{n(1 / p-1), \infty}}=\sup _{\substack{x \in \mathbf{R}^{\mathbf{n}} \\ h \in \mathbf{R}^{\mathbf{n}} \backslash\{0\}}} \frac{\left|\left(\Delta_{h}^{k} f\right)(x)\right|}{|h|^{n(1 / p-1)}},
$$

where, $k \in \mathbf{N}, k>n(1 / p-1)$ and

$$
\left(\Delta_{h}^{k} f\right)(x)=\sum_{r=0}^{k}\left(\begin{array}{l}
k \\
r
\end{array}\right)(-1)^{r} f(x+r h),
$$

is the $k$ th order difference operator. Now, we have the following

LEMMA 22. Fix $y \in \mathbf{R}^{\mathbf{n}}$ and $\alpha>0$. Then

$$
\left\|e^{-i y \delta}\right\|_{\dot{B}_{\infty}^{\alpha, \infty}} \approx|y|^{\alpha} \text {. }
$$

Proof. Let $k \in \mathbf{N}, k>\alpha$ and suppose $y \in \mathbf{R}^{\mathbf{n}} \backslash\{0\}$. Then, for $h \in \mathbf{R}^{\mathbf{n}}$

$$
\begin{aligned}
\left(\Delta_{h}^{k} e^{-i y \cdot}\right)(x) & =\sum_{r=0}^{k}\left(\begin{array}{l}
k \\
r
\end{array}\right)(-1)^{r} e^{-i y(x+r h)} \\
& =e^{-i y x} \sum_{r=0}^{k}\left(\begin{array}{l}
k \\
r
\end{array}\right)(-1)^{r} e^{-i r y h}=e^{-i y x}\left(1-e^{-i y h}\right)^{k} .
\end{aligned}
$$


Hence,

$$
\left|\left(\Delta_{h}^{k} e^{-i y \cdot}\right)(x)\right|^{2}=(2-2 \cos (y h))^{k}
$$

Thus,

$$
\sup _{\substack{x \in \mathbf{R}^{\mathbf{n}} \\ h \in \mathbf{R}^{\mathbf{n}} \backslash\{0\}}} \frac{\left|\left(\Delta_{h}^{k} e^{-i y \cdot}\right)(x)\right|}{|h|^{\alpha}}=\sup _{\substack{h \in \mathbf{R}^{\mathbf{n}} \backslash\{0\}\\}} 2^{k / 2} \frac{(1-\cos (y h))^{k / 2}}{|h|^{\alpha}}
$$

$$
\begin{aligned}
& \leq C_{k}|y|^{\alpha} \sup _{u \in \mathbf{R}^{+}} \frac{(1-\cos u)^{k / 2}}{u^{\alpha}} \\
& \leq C_{k} \sup _{u \in \mathbf{R}^{+}} \frac{(1-\cos u)^{\alpha / 2}}{u^{\alpha}}(1-\cos u)^{(k-\alpha) / 2}|y|^{\alpha} \\
& \leq C_{k, \alpha}|y|^{\alpha},
\end{aligned}
$$

since $k>\alpha$. Conversely, we want to show that for any $y \in \mathbf{R}^{\mathbf{n}} \backslash\{0\}$, there exists an $h \in \mathbf{R}^{\mathbf{n}} \backslash\{0\}$ such that $|y|=|h|^{-1}$ and $1-\cos (y h)=$ $1-\cos (1)>0$. In fact, if $h=y /|y|^{2}$ then trivially $|y|=|h|^{-1}$ and $y \cdot h=1$. Hence

$$
\left\|e^{-i y \cdot}\right\|_{\dot{B}_{\infty}^{\alpha, \infty}} \geq 2^{k / 2}(1-\cos 1)^{k / 2}|y|^{\alpha} .
$$

Thus, by the duality between $H^{p}$ and $\dot{B}_{\infty}^{n(1 / p-1), \infty}, 0<p<1$, and using this lemma, we find that if $g \in \mathscr{S}_{0}$

$$
\begin{aligned}
|\hat{g}(y)| & =\left|\int_{\mathbf{R}^{\mathbf{n}}} g(x) e^{-i y x} d x\right| \leq\|g\|_{H^{p}}\left\|e^{-i y \cdot}\right\|_{\dot{B}_{\infty}^{n(1 / p-1), \infty}} \\
& \leq C_{n, p}|y|^{n(1 / p-1)}\|g\|_{H^{p}} .
\end{aligned}
$$

As a curiosity, and from the proof of Corollary 20, we see that

$$
\left\|e^{-i y \cdot}\right\|_{\dot{B}_{\infty}^{\alpha, \infty}} \approx\left(\int_{0}^{\infty} \widehat{P}_{t}(y) t^{\alpha-1} d t\right)^{-1}, \quad \alpha>0 .
$$

One can also get very easily that, for $s>0,1<q \leq \infty$ we have for the Besov space $\dot{B}_{\infty}^{s, q},\left\|e^{-i y \cdot}\right\|_{\dot{B}_{\infty}^{s, q}} \approx|y|^{s}$. Hence (see [13]), since

$$
\begin{aligned}
&\left(\dot{B}_{p}^{s, q}\right)^{*}=\dot{B}_{\infty}^{-s+n(1 / p-1), q^{\prime}}, \\
& 0<p \leq 1,0<q<\infty, 0<s<n(1 / p-1),
\end{aligned}
$$

and

$$
\begin{aligned}
\left(\dot{F}_{p}^{s, q}\right)^{*}=\dot{B}_{\infty}^{-s+n}(1 / p-1), \infty \\
0<p<1,0<q<\infty, 0<s<n(1 / p-1)
\end{aligned}
$$


where $q^{\prime}=\infty$ if $0<q \leq 1$, and $\dot{F}_{p}^{s, q}$ is a Triebel-Lizorkin space (see [13]), then, by a similar argument as above, we obtain

$$
\begin{aligned}
& |\hat{f}(y)| \leq C|y|^{-s+n(1 / p-1)}\|f\|_{\dot{B}_{p}^{s, q}}, \\
& \quad 0<p \leq 1,0<q<\infty, 0<s<n(1 / p-1),
\end{aligned}
$$

and

$$
\begin{aligned}
& |\hat{f}(y)| \leq C|y|^{-s+n(1 / p-1)}\|f\|_{\dot{F}_{p}^{s, q}} \\
& \quad 0<p<1,0<q<\infty, 0<s<n(1 / p-1) .
\end{aligned}
$$

The following result gives the regularity of a harmonic extension in the $x$-variable, when integrated against an $M^{\alpha}$ measure on $t$.

Corollary 23. Suppose $1 \leq q \leq \infty, \alpha \geq n / q$ and $1 / p=\alpha / n+$ $1 / q^{\prime}$. For a function $f \in L^{q}\left(\mathbf{R}^{\mathbf{n}}\right)$ and $\sigma \in M^{\alpha}$ define

$$
K(f, \sigma)(y)=\int_{0}^{\infty}\left(P_{t} * f\right)(y) d \sigma(t) .
$$

(i) If $0<p<1$ then,

$$
K: L^{q}\left(\mathbf{R}^{\mathbf{n}}\right) \times M^{\alpha} \rightarrow \dot{B}_{\infty}^{n(1 / p-1), \infty},
$$

and

$$
\|K(f, \sigma)\|_{\dot{B}_{\infty}^{n(1 / p-1), \infty}} \leq C_{n}\|\sigma\|_{M^{\alpha}}\|f\|_{L^{q}\left(\mathbf{R}^{\mathbf{n}}\right)} .
$$

(ii) If $p=1$, then

$$
K: L^{q}\left(\mathbf{R}^{\mathbf{n}}\right) \times M^{\alpha} \rightarrow \mathrm{BMO},
$$

and

$$
\|K(f, \sigma)\|_{\mathrm{BMO}} \leq C_{n}\|\sigma\|_{M^{\alpha}}\|f\|_{L^{q}\left(\mathbf{R}^{\mathrm{n}}\right)}
$$

Proof. We will only show (i), because the proof of (ii) follows similarly. Since $\left(H^{p}\left(\mathbf{R}^{\mathbf{n}}\right)\right)^{*}=\dot{B}_{\infty}^{n(1 / p-1), \infty}$, then to show that $K(f, \sigma) \in$ $\dot{B}_{\infty}^{n(1 / p-1), \infty}$ we only need to see that

$$
\left|\int_{\mathbf{R}^{\mathbf{n}}} g(y) K(f, \sigma)(y) d y\right| \leq C_{n}\|\sigma\|_{M^{\alpha}}\|f\|_{L^{q}}\|g\|_{H^{p}},
$$

for all $g \in \mathscr{S}_{0}$. Set $F(z, x)=f(x)$, for all $z \in \mathbf{R}^{\mathbf{n}}$. Then,

$$
\begin{aligned}
\int_{\mathbf{R}^{\mathbf{n}}} g(y) K(f, \sigma)(y) d y & =\int_{\mathbf{R}^{\mathbf{n}}} g(y) \int_{0}^{\infty}\left(P_{t} * F_{z}\right)(y) d \sigma(t) d y \\
& =T_{F}(g, \sigma)(z)
\end{aligned}
$$


for all $z \in \mathbf{R}^{\mathbf{n}}$. Hence, by Theorem 19,

$$
\left|\int_{\mathbf{R}^{\mathbf{n}}} g(y) K(f, \sigma)(y) d y\right| \leq C_{n}\|\sigma\|_{M^{\alpha}}\|f\|_{L^{q}}\|g\|_{H^{p}}
$$

We now give another application of our duality techniques to estimate harmonic extensions to $\mathbf{R}_{+}^{\mathbf{n}+1}$ of functions in $H^{p}$. The next theorem gives, as a particular case, a generalization to higher dimensions of the Féjer-Riesz inequality (see [5] Theorems I-4.5 and III-7.57, for the case $p=1$ ), and shows that it can also be proved in all cases $0<p \leq 1$. Moreover, in the previous theorems, the authors work with the atomic characterization of $H^{1}$ and some extra conditions on the kernel are required, that will not be needed in our proof. This inequality gives the behaviour in the vertical $t$-direction for the extension $\varphi_{t} * f(x)$, relative to a kernel $\varphi$, with $f \in \mathscr{S}_{0}$, instead of the well-known growth on the $x$-direction for the harmonic extension $u \equiv P I(f)$; namely,

$$
\sup _{t>0} \int_{\mathbf{R}^{\mathbf{n}}}|u(x, t)|^{p} d x \leq C\|f\|_{H^{p}}^{p} .
$$

The proof is based in finding the right pairing for an appropriate Carleson measure.

TheOREM 24. If $0<p \leq 1, F \in T_{\infty}^{p}$ and $\sigma \in M^{n / p}$, then

$$
\sup _{x \in \mathbf{R}^{\mathrm{n}}} \int_{0}^{\infty}|F(x, t)| d|\sigma|(t) \leq\|\sigma\|_{M^{n / p}}\|F\|_{T_{\infty}^{p}} .
$$

Proof. Fix $x \in \mathbf{R}^{\mathbf{n}}$ and set $d \mu(y, t)=\delta_{x}(y) d \sigma(t)$, where $\delta_{x}$ is the Dirac mass in $\mathbf{R}^{\mathbf{n}}$ at the point $x$. Then $\mu \in V^{1 / p}$ and $\|\mu\|_{V^{1 / p}} \leq$ $\|\sigma\|_{M^{n / p}}$. In fact, since $p \leq 1$, then if $Q$ is a cube in $\mathbf{R}^{\mathbf{n}}$ we have that

$$
|\mu|(\widehat{Q}) \leq\left(\int_{Q} \delta_{x}(y)\right)\left(\int_{0}^{|Q|^{1 / n}} d|\sigma|(t)\right) \leq|Q|^{1 / p}\|\sigma\|_{M^{n / p}}
$$

Therefore, since $\left(T_{\infty}^{p}\right)^{*}=V^{1 / p}$, we get that

$$
\begin{aligned}
\int_{0}^{\infty}|F(x, t)| d|\sigma|(t) & \leq \int_{\mathbf{R}_{+}^{n+1}}|F(y, t)| d|\mu|(y, t) \\
& \leq\|F\|_{T_{\infty}^{p}}\|\mu\|_{V^{1 / p}} \leq\|\sigma\|_{M^{n / p}}\|F\|_{T_{\infty}^{p}} .
\end{aligned}
$$

For the next result we introduce the following notation (see [14]): if $f \in \mathscr{S}_{0}, 0<p \leq 1$ and we choose $\varphi \in L^{1} \cap L^{\infty}, \int_{\mathbf{R}^{\mathbf{n}}} \varphi(x) d x \neq 0$ then we say that $f \in H_{\varphi}^{p}$ if $\|f\|_{H_{\varphi}^{p}}=\left\|\varphi_{t} * f\right\|_{T_{\infty}^{p}}<\infty$. 
CoRollary 25. Let $\varphi$ be as above, $0<p \leq 1$.

(i) (Féjer-Riesz inequality, if $\varphi$ is the Poisson kernel.) If $f \in H_{\varphi}^{p}$, then

$$
\sup _{x \in \mathbf{R}^{\mathrm{n}}} \int_{0}^{\infty}\left|\left(\varphi_{t} * f\right)(x)\right| t^{n / p-1} d t \leq C_{n, p}\|f\|_{H_{\varphi}^{p}} .
$$

(ii) With more generality, if $p \leq q \leq 1$, then for $f \in H_{\varphi}^{p}$ we have

$$
\sup _{x \in \mathbf{R}^{\mathrm{n}}} \int_{0}^{\infty}\left|\left(\varphi_{t} * f\right)(x)\right|^{q} t^{q n / p-1} d t \leq C_{n, p}\|f\|_{H_{\varphi}^{p}}^{q} .
$$

Proof. (i) Consider the function $F(x, t)=\left(\varphi_{t} * f\right)(x)$ and the measure $d \sigma(t)=t^{n / p-1} d t$. Then $F \in T_{\infty}^{p}$ and $\sigma \in M^{n / p}$. Hence, by the previous theorem,

$$
\begin{aligned}
\sup _{x \in \mathbf{R}^{\mathrm{n}}} \int_{0}^{\infty} & \left|\left(\varphi_{t} * f\right)(x)\right| t^{n / p-1} d t \\
& =\sup _{x \in \mathbf{R}^{\mathrm{n}}} \int_{0}^{\infty}|F(x, t)| d|\sigma|(t) \leq C_{n, p}\|f\|_{H_{\varphi}^{p}} .
\end{aligned}
$$

(ii) Let $p \leq q \leq 1$ and consider now the function

$$
F(x, t)=\left|\left(\varphi_{t} * f\right)(x)\right|^{q} .
$$

Then $F \in T_{\infty}^{p / q}$ with $\|F\|_{T_{\infty}^{p / q}}=\|f\|_{H_{\varphi}^{p}}^{q}$. Also, if we set $d \sigma(t)=$ $t^{q n / p-1} d t$ then $\sigma \in M^{q n / p}$ and hence, since $p / q \leq 1$,

$$
\sup _{x \in \mathbf{R}^{\mathrm{n}}} \int_{0}^{\infty}\left|\left(\varphi_{t} * f\right)(x)\right|^{q} t^{q n / p-1} d t \leq C_{n, p}\|F\|_{T_{\infty}^{p / q}}=C_{n, p}\|f\|_{H_{\varphi}^{p}}^{q} .
$$

\section{REFERENCES}

[1] J. Alvarez and M. Milman, Spaces of Carleson measures: duality and interpolation, Ark. Mat., 25 (2) (1987), 155-174.

[2] R. Coifman, Y. Meyer and E. Stein, Some new function spaces and their applications to harmonic analysis, J. Funct. Anal., 62 (1985), 304-335.

[3] P. L. Duren, B. W. Romberg and A. L. Shields, Linear functionals on $H^{p}$ spaces with $0<p<1$, J. Reine Angew. Math., 238 (1969), 32-60.

[4] C. Fefferman and E. Stein, $H^{p}$ spaces of several variables, Acta Math., 129 (1972), 137-193.

[5] J. Garcia-Cuerva and J. L. Rubio de Francia, Weighted Norm Inequalities and Related Topics, Mathematical Studies, vol. 116, North-Holland, 1985.

[6] Han Yongsheng, Certain Hardy-type spaces that can be characterized by maximal functions and variations of the square functions, Ph.D. Thesis, Washington University (1984). 
[7] R. Johnson, Applications of Carleson Measures to Partial Differential Equations and Fourier Multiplier Problems, Proc. Conf. Cortona, Lecture Notes in Math., vol. 992, (1982), pp. 16-72.

[8] A. Nagel and E. Stein, On certain maximal functions and approach regions, Adv. Math., 54 (1984), 83-106.

[9] F. J. Ruiz and J. L. Torrea, $A$ unified approach to Carleson measures and $A_{p}$ weights. II, Pacific J. Math., 120 (1985), 189-197.

[10] J. Soria, Weighted Tent Spaces, Math. Nachr., 155 (1992), 231-256.

[11] E. M. Stein, Singular Integrals and Differentiability Properties of Functions, Princeton University Press, 1970.

[12] M. Taibleson and G. Weiss, The molecular characterization of certain Hardy spaces, Astérisque, 77 (1980), 67-149.

[13] H. Triebel, Theory of Function Spaces, Birkhäuser-Verlag, Basel, Boston and Stuttgart, 1983.

[14] G. Weiss, Some Problems in the Theory of Hardy Spaces, Proc. Sympos. Pure Math., vol. 35, Amer. Math. Soc., Providence, RI, 1979, pp. 189-200.

Received March 17, 1992 and in revised form September 15, 1992.

Universitat Autònoma de Barcelona

08193 Bellatera Barcelona, Spain

AND

UNIVERSITAT DE BARCELONA

08071 BARCELONA, SPAIN 



\section{CONTENTS}

D. Bisch, A note on intermediate subfactors , . , . . . . . . . . . . . . . . . . 201

M. J. Carro and J. Soria, Tent spaces over general approach regions and pointwise

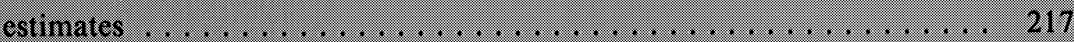

J. A. Charris, M. E. H. Ismail, and S. Monsalve, On sieved orthogonal polynomials X: general blocks of recurrence relations . . . . . . . . . . . . . . . . 237

K. S. Chou and T. Y. H. Wan, Asymptotic radial symmetry for solutions of $\Delta u+$ $e^{\prime \prime}=0$ in a punctured disc . . . . . . . . . . . . . . . . . . . . . . . 269

M. E. Fogel, Knots with algebraic unknotting number one . . . . . . . . . . . . . 277

K. N. Jones, The structure of closed nonpositively curved Euclidean cone 3-manifolds . . . . . . . . . . . . . . . . . . . . . . . . . . . . . . . . 297

M. Kaneda, On the Frobenius morphism of flag schemes . . . . . . . . . . 315

H. T. Kaptanoğlu, Möbius-invariant Hilbert spaces in polydiscs . . . . . . . . . 337

K. Kuribayashi, The cohomology ring of the spaces of loops on Lie groups and homogeneous spaces ............................ 361

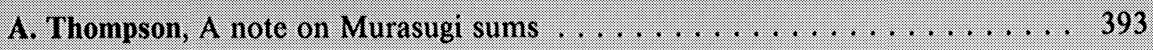




\title{
PACIFIC JOURNAL OF MATHEMATICS
}

\author{
Volume $163 \quad$ No. $2 \quad$ April 1994
}

A note on intermediate subfactors

201

DIETMAR BISCH

Tent spaces over general approach regions and pointwise estimates

217

MARÍA J. CARRO and JAVIER SORIA

On sieved orthogonal polynomials. X. General blocks of recurrence

237 relations

JAIro A. Charris, Mourad ISMAIL and SERGio Monsalve

Asymptotic radial symmetry for solutions of $\Delta u+e^{u}=0$ in a punctured disc

Kai Seng (Kaising) Chou (Tso) and Tom YaU-Heng Wan

Knots with algebraic unknotting number one

MicAh Elton Fogel

The structure of closed non-positively curved Euclidean cone

3-manifolds

KERRY NELSON JONES

On the Frobenius morphism of flag schemes

MASAHARU KANEDA

Möbius-invariant Hilbert spaces in polydiscs

H. TuRgay KaptanOGLU

The cohomology ring of the spaces of loops on Lie groups and homogeneous spaces

KATSUHIKO KURIBAYASHI

A note on Murasugi sums

ABIGAIL A. THOMPSON 